\title{
Color Segmentation Based on Separate Anisotropic Diffusion of Chromatic and Achromatic Channels
}

\author{
Luca Lucchese $e^{\dagger \ddagger}$ and Sanjit K. Mitra \\ ${ }^{\dagger}$ Dept. of Electrical and Computer Eng. \\ University of California at Santa Barbara \\ Santa Barbara, CA 93106 \\ ${ }^{\ddagger}$ Dipartimento di Elettronica e Informatica \\ Università di Padova, Italy \\ Via G. Gradenigo 6/A, 35131 Padova
}

phone: (805) 893 8312, fax: (805) 8933262

e-mail: $\{l u c a, m i t r a\} @ i p l a b . e c e . u c s b . e d u$

\begin{abstract}
This paper presents a new technique for segmenting images only on the basis of color information. It is shown how segmentation can benefit from splitting color signals into chromatic and achromatic channels and separately smoothing them through anisotropic diffusion. Operatively, this is accomplished through two independent diffusion rocesses: one involves only the chromatic information, conveniently embedded in a complex function, while the other affects the lightness information. The results of the two diffusions are separately segmented by $k$-means clustering techniques and their combination yields the final image partition into homogeneous regions. Some experimental results are reported in the paper which verify the effectiveness of the proposed technique.
\end{abstract}

\section{Introduction}

The human visual system (HVS) performs extraordinarily well in spotting out and recognizing the various objects that still or moving pictures portray. On the other side, artificial intelligence systems find it not so straightforward to recognize objects in images, even in the case of simple scenes. In the field of image processing and computer vision, segmentation refers to the low-level operation of partitioning an image into disjoint and homogeneous regions which should be meaningful for a given application; this operation is usually preliminary to higher-level tasks such as object recognition, classification, and semantic interpretation. 
Until a few years ago, segmentation techniques were mainly concerned with gray-level images since for a long time these have been the only kind of visual information that acquisition devices were able to take and computer resources to handle. Rather comprehensive surveys on techniques for segmentation of gray-level images can be found in [1]-[4]. Over the last decade, there has been a remarkable growth of algorithms for segmentation of color images. Among others, two phenomena in particular have triggered such an interest: the recent and fast evolution of the Internet and the parallel development of digital libraries and large databases of images which have been gathering an impressive amount of visual information. Color represents an effective means indeed for indexing and managing this kind of information [5].

An index of the important role played by segmentation of color imagery in a great number of applications may be represented by the almost bewildering variety of techniques advanced to accomplish this task; a few papers provide rather comprehensive surveys on them [6]-[8]. Most of the times, color segmentation techniques are sort of dimensional extensions of analogous techniques devised for gray-level images; they therefore exploit the well-established background laid down in that field. In other cases, they are ad hoc algorithms tailored on the particular nature of color information and on the physics of the interaction of light with colored materials. A classification of color segmentation techniques is proposed in [8] which divides them into three main categories: 1) feature-space based techniques; 2) image-domain based techniques; and 3) physics based techniques.

In this paper we present a new segmentation algorithm belonging to the first category and based on anisotropic diffusion [9, 10] of the chromatic and achromatic components of a color image. This nonlinear filtering technique shows in fact an extremely interesting property from the point of view of segmentation: the smoothing is selective, being encouraged in homogeneous regions and inhibited across region boundaries. Thus, noise and irrelevant image details can be filtered out, making it easier for a segmentation algorithm to achieve spatial compactness while retaining the edge information. Several researchers have resorted to this tool as a preprocessing step for segmentation algorithms operating on scalar images, e.g., $[11,12,13]$. However, even though diffusion of vector-valued functions, such as color images or multispectral data, has already a solid background $[14,15]$, its has been mainly aimed at filtering, denoising, and enhancement. Here we prove the usefulness of anisotropic diffusion also in the context of color image segmentation.

Since color is a three-dimensional (3D) signal, almost all of the available segmentation techniques treat it as such [6]-[8]. In this paper we regard color as a $2 \mathrm{D}+1 \mathrm{D}$ signal by decomposing it into its chromatic and achromatic components which are separately segmented and recomposed in the final result. This new way of tackling the problem of color segmentation finds its rationale in 
the modern theories and models of color vision [17]-[23]: The human visual system senses color information from the outer world through photoreceptors which can be regarded as three sets of filters tuned to the wavelenghts of red, green, and blue; this information is then split into chromatic (2D) and achromatic (1D) channels before being further and independently processed (zone theory, [17]). Neurophysiological evidence shows that there exists a perfect agreement between the second HVS processing stage and the opponent-colors theory suggested by Hering [17] and based on the three antagonistic mechanisms red-green, blue-yellow, and black-white. These stimuli can also be conveniently expressed in terms of hue, saturation, and lightness (or brightness) through an opportune change of reference systems [21, 22]. Such a choice of color percepts is suggested by the fact that hue and saturation are the color features which provide the most useful basis for judging color uniformity, being rather invariant to surface curvature and lighting conditions [24]. A space carrying hue and saturation information is therefore the ideal candidate for chromatic segmentation. On the other hand, lightness is a proper discriminant for segmenting regions with low chromatic content.

In our scheme, hue and saturation are processed together using the formalism of phasors: hue is the phase and saturation is the magnitude of a complex function defined as the complex chromaticity. The diffusion of this complex quantity is shown to be equivalent to a system of coupled diffusion equations affecting hue and saturation with a mutual feedback; in fact, hue and saturation are physically related to each other (hue variations are irrelevant at low saturation and important at high saturation) and this is accomodated for by our formalism. After diffusion, the complex chromaticity is unsupervisedly segmented by means of a clustering algorithm. The scalar achromatic information represented by lightness is separately diffused, clustered, and segmented. The combination of the two parallel segmentation processes allows the original color image to be partitioned. We report a few examples which confirm the effectiveness of this technique.

This work has four sections. Section 2 briefly discusses the formats and the vector spaces used in this paper for representing and handling color information. Section 3 details our segmentation technique. Section 4 finally draws the conclusions.

\section{Color Representation}

We will adopt two different representations of a color image $\mathcal{C}$ depending on whether or not spatial relationships among pixels are important for a certain image processing operation. When they are, we represent $\mathcal{C}$ in a vector form as $\boldsymbol{C}(\boldsymbol{x})=[R(\boldsymbol{x}) G(\boldsymbol{x}) B(\boldsymbol{x})] \in \mathbb{R}^{3}, \boldsymbol{x} \in \mathbb{R}^{2}$, where $R(\boldsymbol{x}), G(\boldsymbol{x})$, and $B(\boldsymbol{x})$ are, respectively, the red, green, and blue channels conveniently normalized between 0 
and 1. Although hue, saturation, and lightness have been introduced in the previous sections with reference to the opponent-colors theory, we may define these three perceptual attributes of color in various other ways. Since our algorithm is based on clustering of chromatic and achromatic information, we would like that these two operations were done in uniform spaces; this suggests the use of hue, saturation, and lightness associated with the $L^{*} u^{*} v^{*}$ color space [24]. We thus express the color image in the $X Y Z$ space as $\boldsymbol{C}(\boldsymbol{x})=[X(\boldsymbol{x}) Y(\boldsymbol{x}) Z(\boldsymbol{x})]$, where [24]

$$
\left[\begin{array}{l}
X(\boldsymbol{x}) \\
Y(\boldsymbol{x}) \\
Z(\boldsymbol{x})
\end{array}\right]=\left[\begin{array}{lll}
0.49000 & 0.31000 & 0.20000 \\
0.17697 & 0.81240 & 0.01063 \\
0.00000 & 0.01000 & 0.99000
\end{array}\right]\left[\begin{array}{l}
R(\boldsymbol{x}) \\
G(\boldsymbol{x}) \\
B(\boldsymbol{x})
\end{array}\right] .
$$

From Eq. (1), the two chromatic channels $u^{\prime}(\boldsymbol{x})$ and $v^{\prime}(\boldsymbol{x})$ are derived, respectively, as

$$
u^{\prime}(\boldsymbol{x})=\frac{4 X(\boldsymbol{x})}{X(\boldsymbol{x})+15 Y(\boldsymbol{x})+3 Z(\boldsymbol{x})} \quad \text { and } \quad v^{\prime}(\boldsymbol{x})=\frac{9 Y(\boldsymbol{x})}{X(\boldsymbol{x})+15 Y(\boldsymbol{x})+3 Z(\boldsymbol{x})} .
$$

This information may be expressed in a polar form through the hue-angle

$$
\vartheta(\boldsymbol{x}) \doteq \arctan \left(\frac{v^{\prime}(\boldsymbol{x})-v_{N}^{\prime}}{u^{\prime}(\boldsymbol{x})-u_{N}^{\prime}}\right)
$$

and the saturation ${ }^{1}$

$$
\sigma(\boldsymbol{x}) \doteq \sqrt{\left(u^{\prime}(\boldsymbol{x})-u_{N}^{\prime}\right)^{2}+\left(v^{\prime}(\boldsymbol{x})-v_{N}^{\prime}\right)^{2}},
$$

where $u_{N}^{\prime}$ and $v_{N}^{\prime}$ are the values of $u^{\prime}$ and $v^{\prime}$ of a suitably chosen reference white [24]. In our experiments, we have adopted the coordinates of the standard illuminant $D_{65}: u_{N}^{\prime}=0.1978$ and $v_{N}^{\prime}=0.4683$. As an example, Fig. 3 (top row) shows ${ }^{2}$ the signals $\vartheta(\boldsymbol{x})$ and $\sigma(\boldsymbol{x})$ relative to the image of the parrots of Fig. 7. With the formalism of phasors, we conveniently represent these chromatic components in the complex plane $\mathbb{C}$ as $\kappa(\boldsymbol{x})=\sigma(\boldsymbol{x}) \exp (j \vartheta(\boldsymbol{x}))$ (analogous representations were also suggested in [26] and in [27]). Henceforth, $\kappa(\boldsymbol{x})$ will be referred to as the complex chromaticity. The values taken in by $\kappa(\boldsymbol{x})$ may be displayed in the chromaticity diagram $u^{\prime} v^{\prime}$ [24], by letting $u^{\prime}=u_{N}^{\prime}+\Re(\kappa(\boldsymbol{x}))$, and $v^{\prime}=v_{N}^{\prime}+\Im(\kappa(\boldsymbol{x}))$, where $\Re($.$) and \Im($.$) respectively denote the real and$ the imaginary part. Fig. 1 (a) shows the $u^{\prime} v^{\prime}$ chromaticity diagram relative to the image of the parrots of Fig. 7 along with the spectral locus and the line of purples (solid line) and the reference white (small circle).

\footnotetext{
${ }^{1}$ For convenience, we dropped the scaling factor 13 usually included in the definition of saturation in $u^{\prime} v^{\prime}$ coordinates [24].

${ }^{2}$ Hue is defined over $[0,2 \pi)$ and is displayed in such a way that 0 corresponds to black and $2 \pi$ to white; similarly, a saturation of value 0 is displayed as black and its maximum value (depending on the image) is set as white.
} 
The achromatic channel is encoded through the CIE $L^{*}$ lightness signal defined as [24]

$$
\ell(\boldsymbol{x})= \begin{cases}116\left(\frac{Y(\boldsymbol{x})}{Y_{N}}\right)^{3}-16, & \text { for } \frac{Y(\boldsymbol{x})}{Y_{N}}>0.008856, \\ 903.3\left(\frac{Y(\boldsymbol{x})}{Y_{N}}\right) & \text { for } \frac{Y(\boldsymbol{x})}{Y_{N}} \leq 0.008856\end{cases}
$$

where $Y_{N}$ is the $Y$ value for some reference white; we have chosen $Y_{N}=1$ (perfect diffuser). Fig. 3 (top row) shows the lightness signal $\ell(\boldsymbol{x})$ relative to the image of the parrots of Fig. 7 .

If spatial information is not essential for a certain task, the color image $\mathcal{C}$ may be represented more compactly in a palettized format. Suppose that $\mathcal{C}$ is available in a digital form as a $M \times N$ matrix, whence the three matrices ${ }^{3} \mathcal{R}, \mathcal{G}$, and $\mathcal{B} \in \mathbb{R}_{[0,1]}^{M \times N}$, are the sampled counterparts of $R(\boldsymbol{x})$, $G(\boldsymbol{x})$, and $B(\boldsymbol{x})$, respectively. The color image can then be expressed as $\mathcal{C}=\{\boldsymbol{\mathcal { Q }}, \mathcal{P}\}$, where $\mathcal{Q} \in \mathbb{N}_{[1, L]}^{M \times N}$ is a matrix of pointers to a look-up-table of colors $\mathcal{P} \in \mathbb{R}_{[0,1]}^{L \times 3}$, with $L$ denoting the number of possible combinations of the entries of $\mathcal{R}, \mathcal{G}$, and $\mathcal{B}$. In the $R G B$ format, the color palette $\mathcal{P}$ may be structured as $\mathcal{P}=[\mathbf{R} \mathbf{G} \mathbf{B}]$, where $\mathbf{R}, \mathbf{G}, \mathbf{B} \in \mathbb{R}_{[0,1]}^{L}$ are, respectively, the vectors containing the red, green, and blue coordinates of the possible combinations of colors within $\mathcal{C}$. Of course, $\mathcal{P}$ may be expressed in other color spaces as well.

In the scheme of Fig. 4, the symbol $\mathcal{T}$ denotes the set of color transformations which split the $R G B$ color signal $\boldsymbol{C}(\boldsymbol{x})$ into the chromatic channel $\kappa(\boldsymbol{x})$ and the achromatic channel $\ell(\boldsymbol{x})$. The inverse of this set of transformations is denoted as $\mathcal{T}^{-1}$.

\section{Segmentation Algorithm}

\subsection{Anisotropic diffusion of the chromatic and achromatic channels}

The complex chromaticity $\kappa(\boldsymbol{x})$ is embedded in a one-parameter family of "derived" images $\kappa(\boldsymbol{x}, t)$, where the parameter $t \in \mathbb{R}$ can be regarded either as time or as an iteration step; more explicitly, this function reads $\kappa(\boldsymbol{x}, t)=\sigma(\boldsymbol{x}, t) \exp (j \vartheta(\boldsymbol{x}, t)) \in \mathbb{C}$. The anisotropic diffusion of $\kappa(\boldsymbol{x}, t)$ is carried out by means of the partial differential equation [9]

$$
\frac{\partial}{\partial t} \kappa(\boldsymbol{x}, t)=\operatorname{div}(c(\boldsymbol{x}, t) \nabla \kappa(\boldsymbol{x}, t))
$$

where $\operatorname{div}$ and $\nabla \doteq\left[\frac{\partial}{\partial x} \frac{\partial}{\partial y}\right]^{T}$ respectively denote the divergence and the gradient operators, and $c(\boldsymbol{x}, t)=f(|\nabla \kappa(\boldsymbol{x}, t)|)$ is a monotonically decreasing function of the image gradient magnitude

\footnotetext{
${ }^{3}$ Throughout the text, the notation $\boldsymbol{A} \in \mathbb{S}_{[\alpha, \beta]}^{M \times N}$ indicates that each element $a_{i j}$ of the matrix $\boldsymbol{A} \in \mathbb{S}^{M \times N}$ takes in values in the set $[\alpha, \beta] \subset \mathbb{S}$.
} 
(conductance coefficient), chosen [9] as

$$
c(\boldsymbol{x}, t)=\left(1+\left(\frac{|\nabla \kappa(\boldsymbol{x}, t)|}{\gamma_{\kappa}}\right)^{2}\right)^{-1} .
$$

Between the two conductance coefficients usually adopted [9], the form in Eq. (7) has been preferred because it tends to favor wider regions over smaller ones (the other is expected instead to favor highcontrast edges over low-contrast ones); for segmentation applications, this certainly is a desirable feature. In the scheme of Fig. 4, the anisotropic diffusion operation carried out on $\kappa(\boldsymbol{x})$ is denoted as $\mathcal{A D}(\mathbb{C})$.

The gradient of $\kappa(\boldsymbol{x}, t)$ (chromatic gradient) is found to be

$$
\nabla \kappa(\boldsymbol{x}, t)=(\nabla \sigma(\boldsymbol{x}, t)+j \sigma \nabla \vartheta(\boldsymbol{x}, t)) \exp (j \vartheta(\boldsymbol{x}))
$$

and its magnitude is $|\nabla \kappa(\boldsymbol{x}, t)|=\left(\|\nabla \sigma(\boldsymbol{x}, t)\|^{2}+\sigma^{2}(\boldsymbol{x}, t)\|\nabla \vartheta(\boldsymbol{x}, t)\|^{2}\right)^{1 / 2}$. It should be noted that, in the magnitude of the chromatic gradient, variations of hue are correctly weighted by the level of color saturation, in agreement with the fact that hue becomes less important at low saturation.

The selective smoothing of the nonlinear filter implemented by Eq. (6) is highly dependent, through Eq. (7), on the diffusion constant $\gamma_{\kappa}$. As pointed out in [9], smoothing within regions is encouraged by $c \simeq 1$ and smoothing inhibition across boundaries is achieved with $c \simeq 0$. Various heuristic strategies have been suggested to select such a parameter, which can be either kept constant during the diffusion process or set as time-dependent [9]. In our implementation, we have preferred the second strategy and dynamically selected $\gamma_{\kappa}$ as the $5 \%$ of the maximum value ${ }^{4}$ of $|\nabla \kappa(\boldsymbol{x}, t)|$ at each iteration. For the numerical implementation of Eq. (6), we have followed the simple discretization scheme provided by Perona and Malik in [9] with $N_{i}=30$ iterations. We denote as $\kappa_{d}(\boldsymbol{x}) \doteq \sigma_{d}(\boldsymbol{x}) \exp \left(j \vartheta_{d}(\boldsymbol{x})\right)$ the result of the diffusion process of $\kappa(\boldsymbol{x}, t)$ at time $t=N_{i}$. Fig. 3 shows $\vartheta_{d}(\boldsymbol{x})$ and $\sigma_{d}(\boldsymbol{x})$ relative to $\vartheta(\boldsymbol{x})$ and $\sigma(\boldsymbol{x})$ of the same figure.

A few important observations are in order. First, the formalism of complex phasors that we have adopted to model the chromatic information of images allows us to carry out the diffusion of an intrinsically vector-valued function in a "scalar" fashion, without resorting to ad hoc techniques for vector-valued images [14]. Second, the combined diffusion of hue and saturation through $\kappa(\boldsymbol{x})$ allows us to bypass the possible numerical instabilities associated with the diffusion of hue alone [28]. Third, the beneficial synergism of hue and saturation components in the diffusion process of Eq. (6), which is the main contribution of this work, may be better appreciated by separating the

\footnotetext{
${ }^{4}$ The hue function $\vartheta(\boldsymbol{x})$ takes in values in $[0,2 \pi)$; however, accounting for the circular nature of this quantity, the difference between $\vartheta_{1}$ and $\vartheta_{2}$ is defined to be $\vartheta_{1}-\vartheta_{2} \doteq \min (\Delta \vartheta, 2 \pi-\Delta \vartheta)$ where $\Delta \vartheta \doteq\left|\vartheta_{1}-\vartheta_{2}\right|$.
} 
real and imaginary parts of such equation. By doing so, we get

$$
\left\{\begin{array}{l}
\frac{\partial}{\partial t} \sigma=\operatorname{div}(c \nabla \sigma)-c \sigma\|\nabla \vartheta\|^{2}, \\
\frac{\partial}{\partial t} \vartheta=\operatorname{div}(c \nabla \vartheta)+2\left(\frac{c}{\sigma}\right) \nabla^{T} \sigma \nabla \vartheta,
\end{array}\right.
$$

where we have dropped space and time variables for compactness. Based upon the system of coupled diffusion equations in Eq. (9), we can make the following remarks. 1) $\|\nabla \vartheta\| \simeq 0$ in a region where hue is almost constant; hue and saturation tend therefore to diffuse independently and the common conductance coefficient depends only on the saturation gradient information because $c \simeq f(|\nabla \sigma|)$. 2) $\|\nabla \sigma\| \simeq 0$ in a region where saturation is almost constant; in this case the conductance coefficient depends on the hue gradient information weighted by saturation since $c \simeq f(\sigma|\nabla \vartheta|)$. The hue diffusion depends on saturation information only through the conductance coefficient: the diffusion is inhibited by high saturation values. Instead, the saturation diffusion shows a negative feedback, weighted by saturation itself, with the hue gradient; this means that a hue edge tends to contrast the saturation diffusion and that this action is more effective at high levels of saturation. 3) In a region where both hue and saturation are almost constant, $c \simeq 1$ and the two chromatic components are decoupled and diffuse independently at high rate.

The lightness signal $\ell(\boldsymbol{x})$ is diffused with an analogous procedure; the signal is embedded in a one-parameter family of "derived" images $\ell(\boldsymbol{x}, t)$ and filtered with Eqs. (6) and (7) by replacing $\kappa(\boldsymbol{x})$ with $\ell(\boldsymbol{x})$. In this case, the value of the diffusion constant $\gamma_{\ell}$ is set as the $5 \%$ of $|\nabla \ell(\boldsymbol{x})|$ at each iteration; the number of iterations is again $N_{i}=30$. Fig. 3 shows the diffused lightness $\ell_{d}(\boldsymbol{x})$ relative to $\ell(\boldsymbol{x})$ of the same figure. In the scheme of Fig. 4, the anisotropic diffusion operation carried out on $\ell(\boldsymbol{x})$ is denoted as $\mathcal{A D}(\mathbb{R})$. The selective smoothing ${ }^{5}$ is apparent in all three diffused signals of Fig. 3 (second row).

It is worth mentioning that recently Tang and Sapiro [29] have proposed an approach very similar to ours and aimed at image denoising: color data are in fact separated into chromaticity and brightness and then indipendently processed with partial differential equations or diffusion flows.

\footnotetext{
${ }^{5}$ By comparing the signals $\vartheta(\boldsymbol{x})$ and $\vartheta_{d}(\boldsymbol{x})$ in Fig. 3, one might think that the white regions of the former signal have been somehow "lost" in the latter; however, as pointed out in Section 2, hue is defined upon a unit circle whence very dark gray shades (associated with $\vartheta \in[0, \epsilon], \epsilon<<2 \pi$ ) are contiguous to very light gray shades (associated with $\vartheta \in[2 \pi-\epsilon, 2 \pi))$.
} 


\subsection{Quantization}

The diffusion process, in general, produces as many values in $\vartheta_{d}(\boldsymbol{x}), \sigma_{d}(\boldsymbol{x})$, and $\ell_{d}(\boldsymbol{x})$ as the product of the image dimensions, and clustering based on these functions would be overly lengthy [30]. Quantization thus represents a useful means for reducing the number of values in the hue, saturation, and lightness signals. Since a separate quantization of the chromatic and achromatic channels might introduce color shifts once the two channels are eventually recombined and transformed into $R G B$ coordinates, a color quantization directly in the $R G B$ space is recommended.

Among the most popular quantization algorithms are the splitting algorithms [31, 32] that divide the $R G B$ cube into disjoint regions and select a representative color from each region as a palette color; they differ in the methods adopted for splitting the color space. The minimum variance quantization method proposed by Heckbert [31] cuts the color cube in the red, green, and blue directions until a chosen number of non-empty regions is obtained; it then uses the average color in each region to create the new reduced palette. We have resorted to this algorithm which can be found already implemented in many software packages for digital image processing applications.

Operatively, we proceed as follows: 1) By inverting the transformation relationships reported in Section 2, from $\vartheta_{d}(\boldsymbol{x}), \sigma_{d}(\boldsymbol{x})$, and $\ell_{d}(\boldsymbol{x})$ we construct the diffused $R G B$ image $\boldsymbol{C}_{d}(\boldsymbol{x})=$ $\left[R_{d}(\boldsymbol{x}) G_{d}(\boldsymbol{x}) B_{d}(\boldsymbol{x})\right]$. 2) We then quantize $\boldsymbol{C}_{d}(\boldsymbol{x})$ as $\boldsymbol{C}_{q}(\boldsymbol{x})$ with $N_{q}$ colors (e.g., $\left.N_{q}=1024\right)$. In the scheme of Fig. 4, the quantization of $\boldsymbol{C}_{d}(\boldsymbol{x})$ into $\boldsymbol{C}_{q}(\boldsymbol{x})$ is denoted by the symbol $\mathcal{Q}$. 3) Finally, we represent $\boldsymbol{C}_{q}(\boldsymbol{x})$ in a palettized format as $\left\{\mathcal{Q}_{q}, \boldsymbol{P}_{q}, \boldsymbol{w}_{q}\right\}$, where ${ }^{6}$ the values taken in by $\mathcal{Q}_{q}$ are pointers to a look-up-table $\boldsymbol{P}_{q}=\left[\begin{array}{ll}\boldsymbol{\kappa}_{q} & \boldsymbol{\ell}_{q}\end{array}\right]$ (a matrix of size $N_{q} \times 2$ with complex entries in the first column and real entries in the second), $\boldsymbol{\kappa}_{q} \doteq \boldsymbol{\sigma}_{q} \exp \left(j \boldsymbol{\vartheta}_{q}\right) \in \mathbb{C}^{N_{q}}$, and $\boldsymbol{w}_{q}$ is a vector that contains the number of pixels in $\mathcal{Q}_{q}$ per each value of $\boldsymbol{P}_{q}$. As an example, Fig. 2 portrays the quantized chromaticities $\boldsymbol{\kappa}_{q}$ relative to the quantization of the chromaticities of Fig. 1 (a).

\subsection{Estimation of the chromatic spreading}

The distribution of the complex chromaticities in the $u^{\prime} v^{\prime}$ plane is image-dependent; hence, the spreading of these points has to be somehow estimated in order to tailor on it the parameters of the following clustering stage. To this end, in [16] we have suggested the following statistical criterion. Let us consider the probability function $F(x) \doteq P\left[\left\|\kappa-\kappa_{\beta}\right\| \leq \xi\right], \xi \geq 0$, which describes the distribution of the distances between each chromaticity $\kappa$ and the baricenter $\kappa_{\beta}$ of the data. If the chromaticities were Gaussian distributed around $\kappa_{\beta}$, the probability density function $f(\xi) \doteq$ $d F(\xi) / d \xi$ would be the Rayleigh density function [33] $f(\xi)=\left(\xi / \sigma^{2}\right) e^{-\xi^{2} / 2 \sigma^{2}} u(\xi)$, where $u(\xi)$ is the

\footnotetext{
${ }^{6}$ Multiplication and exponentiation in the complex vector $\boldsymbol{\kappa}_{q}$ are to be regarded as element-wise operations.
} 
unit-step function. Albeit real imagery usually does not give rise to Gaussian distributed points in the chromaticity plane, we could ascertain experimentally that a Rayleigh density function may describe the statistical distribution of the data fairly well.

We observe that the statistical description above is derived from the original image and not from its diffused and quantized version because experimentally we could notice that, even if the point spreading in the relative $u^{\prime} v^{\prime}$ diagrams looks very similar, a Rayleigh pdf modeling of their distances to the baricentric point suits the first data better than the second.

Our algorithm computes the (discrete) distance function $d(l) \doteq\left\|\kappa(l)-\kappa_{\beta}\right\|, l=1, \ldots, L$, and fits its frequency histogram $\mathcal{H}$ (we used 50 bins) with the continuous function $g(\xi)=\nu f(\xi)$, where the coefficient $\nu$ accounts for the bias of the data; the fitting is performed via a standard non-linear least squares algorithm. Fig. 1 (b) reports the function $g(\xi)$ (solid line) along with the frequency histogram $\mathcal{H}$ (stemmed plot) for the complex chromaticities of Fig. 1 (a). Figs. 5 and 6 show two color images with their palettes, their $u^{\prime} v^{\prime}$ chromaticity diagrams, the relative histograms $\mathcal{H}$, and the Rayleigh pdf's $g(\xi)$ describing the relative chromatic dispersion. In all cases, the appropriateness of this statistical model is apparent.

The probability function $f(\xi)$ allows us to estimate the radius $\varrho_{\eta}$ of the circle in which a certain percentage $\eta$ of the data $(0 \leq \eta \leq 1)$ is contained; such radius is given by $\varrho_{\eta}=\sigma \sqrt{2 \ln (1 /(1-\eta))}$ and represents an index of the chromatic dispersion of the data (in our experiments, we have chosen $\eta=0.95)$.

\subsection{Complex chromaticity clustering and segmentation}

The points in the $u^{\prime} v^{\prime}$ space that, after the quantization step of Section 3.2, have been conveniently represented as a single vector $\boldsymbol{\kappa}_{q} \in \mathbb{C}^{N_{q}}$ need now to be clustered; the literature of pattern recognition offers a number of different techniques for this task. We have employed the well-known $k$-means algorithm [34], whose implementation for our application is reported next [16]. In Fig. 4, the $k$-means algorithm applied to $\boldsymbol{\kappa}_{q}$ has been denoted as $\mathcal{K}(\mathbb{C})$.

\section{$\mathcal{K}(\mathbb{C}):$ The $\mathrm{k}-\mathrm{means}$ algorithm for clustering $\boldsymbol{\kappa}_{q}$}

$\triangleright$ compute the baricenter of the complex chromaticities $\boldsymbol{\kappa}_{q}$ (see Section 3.2) as $\kappa_{q \beta}=\boldsymbol{w}_{q}^{T} \boldsymbol{\kappa}_{q}$;

$\triangleright$ sort the vector $\boldsymbol{w}_{q}$ in descending order and define the vector $\boldsymbol{p} \in \mathbb{N}_{\left[1, N_{q}\right]}^{N_{q}}$ containing such ordering; 
$\triangleright$ set $\kappa_{q \beta}$ as the initial seed for clustering data in the $u^{\prime} v^{\prime}$ space, associate it with a weight $w_{q \beta}=0$, and initialize with them, respectively, two ordered sets $\Omega \subset \mathbb{C}$ and $W_{\omega} \subset \mathbb{Q}_{[0,1]}$ whose elements are dinamically ordered according to their insertion or extraction order;

for $n=1$ to $n=N_{q}$

$\triangleright$ compute the minimum distance $d(i)$ between $\boldsymbol{\kappa}_{\boldsymbol{q}}(n)$ and the cluster seeds in the set $\Omega$ as $d(i)=\min _{\boldsymbol{\omega} \in \Omega}\left\|\boldsymbol{\kappa}_{\boldsymbol{q}}(n)-\boldsymbol{\omega}\right\| ;$ let $\boldsymbol{\omega}(i) \in \Omega$ denote the closest seed;

if $d(i)>\tau_{1}$

then

$\triangleright$ add $\boldsymbol{\kappa}_{\boldsymbol{q}}(n)$ to the set $\Omega$ as a new seed and its associated weight $\boldsymbol{w}(\boldsymbol{p}(n))$ to the set $W_{\omega}$;

else

$\triangleright \boldsymbol{\kappa}_{\boldsymbol{q}}(n)$ belongs to the cluster defined by $\boldsymbol{\omega}(i)$; this induces the shifting of the seed $\boldsymbol{\omega}(i)$ whose new position and associated weight are respectively updated as

$$
\boldsymbol{\omega}(i):=\frac{w_{\boldsymbol{\omega}}(i) \boldsymbol{\omega}(i)+\boldsymbol{w}(\boldsymbol{p}(n)) \boldsymbol{\kappa}_{\boldsymbol{q}}(n)}{w_{\boldsymbol{\omega}}(i)+\boldsymbol{w}(\boldsymbol{p}(n))} \quad \text { and } \quad w_{\boldsymbol{\omega}}(i):=w_{\boldsymbol{\omega}}(i)+\boldsymbol{w}(\boldsymbol{p}(n)) ;
$$

$\triangleright$ compute the minimum distance $d(j)$ between $\boldsymbol{\omega}(i)$ and the other seeds in $\Omega$ as $d(j)=$

$$
\begin{aligned}
& \min _{\substack{\boldsymbol{\omega} \in \Omega \\
\boldsymbol{\omega} \neq \boldsymbol{\omega}(i)}}\|\boldsymbol{\omega}(i)-\boldsymbol{\omega}\| ; \\
& \text { if } d(j)<\tau_{2}
\end{aligned}
$$

then

$\triangleright$ the clusters associated with the seeds $\boldsymbol{\omega}(i)$ and $\boldsymbol{\omega}(j)$ must be merged together and the position and weight of the new resulting cluster are computed according to the following procedure:

$\triangleright$ define $m \doteq \min (i, j)$ and $M \doteq \max (i, j)$;

$\triangleright$ update

$$
\boldsymbol{\omega}(m):=\frac{w_{\boldsymbol{\omega}}(i) \boldsymbol{\omega}(i)+w_{\boldsymbol{\omega}}(j) \boldsymbol{\omega}(j)}{w_{\boldsymbol{\omega}}(i)+w_{\boldsymbol{\omega}}(j)} \quad \text { and } \quad w_{\boldsymbol{\omega}}(m):=w_{\boldsymbol{\omega}}(i)+w_{\boldsymbol{\omega}}(j) ;
$$

$\triangleright$ remove the seed $\boldsymbol{\omega}(M)$ from $\Omega$ and its weight $w_{\boldsymbol{\omega}}(M)$ from $W_{\omega}$;

end

end

end

Some comments are here in order. First of all, the above algorithm requires only one iteration; the number of clusters changes during the clustering process and we do not know a priori the number of clusters we will end up with. When a point must be associated with a cluster or two 
cluster seeds must be merged together, a center of gravity law is adopted; in this way, the outcoming seed is closer to the "heaviest" of the two points, that is, to the point which, in the chromaticity plane, represents the color entering the highest percentage of pixels. Moreover, our procedure spans the points from the "heaviest" to the "lightest" so that, after the first iterations, the seeds do not move significantly, since the early formed seeds are rather "heavy." The fact that we choose only one initial seed instead of many does not affect the performance of the algorithm [34].

On the contrary, the clustering process is affected by the two parameters $\tau_{1}$ and $\tau_{2}$ which respectively determine the inter-cluster and intra-cluster dispersions; they are to be properly selected since they determine to a broad extent the final number of phases in the segmented image. Since a good measure of the chromatic dispersion within the $u^{\prime} v^{\prime}$ plane is provided by $\varrho_{\eta}$, it is reasonable to define $\tau_{1}$ and $\tau_{2}$ as fractions of such parameter. A good example of this adaptivity can be seen by comparing Figs. 5 and 6 : the smaller $\varrho_{\eta}$, the smaller the inter- and intra-cluster parameters. Experimentally, we have found that setting $\tau_{1}=\varrho_{\eta} / 2$ and $\tau_{2}=\varrho_{\omega} / 4$ gives good segmentation results with a wide variety of color images; this choice produces a limited number of segments (usually, less than 20). In the case of Fig. 3, the choice of parameters suggested above has returned the six clusters $\boldsymbol{\omega}(n)=\boldsymbol{\sigma}_{c}(n) \exp \left(j \boldsymbol{\vartheta}_{c}(n)\right), n=1, \ldots, N_{\omega}=6$ shown with crosses in Fig. 2. Instead, $N=11$ clusters were found in the case of Fig. 5 and $N=20$ clusters in the case of Fig. 6 (they are represented with red "+" symbols).

Of course, one might decide to reduce (increase) by supervision the number of segments by increasing (reducing) $\tau_{1}$, always as a fraction of $\varrho_{\eta}$; the parameter $\tau_{2}$ has to be accordingly modified with the only constraint that $\tau_{2} \leq \tau_{1} / 2$ to prevent cluster overlapping.

The segmentation $\mathcal{S}(\mathbb{C})$ (see Fig. 4) of the chromatic channel is finally simply obtained as follows. At each pixel, the diffused chromaticity $\kappa_{d}$ is now replaced by the nearest among the $N_{\omega}$ clusters $\boldsymbol{\omega}(n)$ according to $\kappa_{s}=\arg \min _{\boldsymbol{\omega}(n) \in \Omega}\left|\kappa_{d}-\boldsymbol{\omega}(n)\right|$. The result of such operation is a segmented complex chromaticity $\kappa_{s}(\boldsymbol{x}) \doteq \sigma_{s}(\boldsymbol{x}) \exp \left(j \vartheta_{s}(\boldsymbol{x})\right)$; Fig. 3 shows the segmented versions ${ }^{7} \sigma_{s}(\boldsymbol{x})$ and $\vartheta_{s}(\boldsymbol{x})$ of the respective diffused signals of the same figure.

\subsection{Lightness clustering and segmentation}

The achromatic channel undergoes a similar processing. The same algorithm $\mathcal{K}(\mathbb{C})$ with opportune modifications is used for clustering the lightness $\boldsymbol{\ell}_{q}$ (in Fig. 4 , this operation is referred to as

\footnotetext{
${ }^{7} \mathrm{~A}$ median filter with size $3 \times 3$ was applied to $\sigma_{s}(\boldsymbol{x})$ and $\vartheta_{s}(\boldsymbol{x})$ to remove isolated outliers; in fact, anisotropic diffusion preserves image features with high gradient values so that not only edges but also some noise, e.g. salt-andpepper noise, can be preserved.
} 
$\mathcal{K}(\mathbb{R}))$; the thresholds $\tau_{3}$ and $\tau_{4}$, which respectively play the $1 \mathrm{D}$ counterpart of $\tau_{1}$ and $\tau_{2}$, have been experimentally chosen as $\tau_{3}=20$ and $\tau_{4}=10$. In fact, only a few levels of lightness are required for a good segmentation and with our choice of parameters usually we obtain 2 to 5 regions. In fact, as $\tau_{3}$ determines the inter-cluster distance, it has been set equal to half the scale range in order not to generate too many segments; $\tau_{4}$ determines instead the intra-cluster spreading and it has to satisfy the constraint $\tau_{4} \leq \tau_{3} / 2$ to prevent cluster overlapping. However, the selection of such 1D thresholds is not so crucial as that of the $2 \mathrm{D}$ thresholds, since rather wide changes of $\tau_{3}$ and $\tau_{4}$ yield not appreciably different segmentation results. This choice give $N_{\omega_{\ell}}=3$ clusters, $\boldsymbol{\omega}_{\ell}(1)=54.1467$, $\boldsymbol{\omega}_{\ell}(2)=67.9022$, and $\boldsymbol{\omega}_{\ell}(3)=85.8514$ (the maximum value of $\ell(\boldsymbol{x})$ in Fig. 3 is 100). Similarly to the above, the replacement of the lightness $\ell_{d}$ at each pixel with the closest of the $N_{\omega_{\ell}}$ clusters is carried out through $\ell_{s}=\arg \min _{\boldsymbol{\omega}_{\ell}(n)}\left|\ell_{\boldsymbol{d}}-\boldsymbol{\omega}_{\ell}(n)\right|$ and results in the segmented lightness $\ell_{s}(\boldsymbol{x})$. This operation is denoted as $\mathcal{S}(\mathbb{C})$ in Fig. 4. Fig. 3 shows the segmented version $\ell_{s}(\boldsymbol{x})$ relative to the diffused lightness of the same figure.

\subsection{Final segmentation and discussion}

By combining the segmentations of hue, saturation, and lightness of the last row of Fig. 3 and converting back the chromatic and achromatic channels into $R G B$ coordinates (operation denoted as $\mathcal{T}^{-1}$ in Fig. 4), we finally obtained the result shown in Fig. 7. Figs. 7 and 8 also report other examples of segmentation with various types of images which bear out the effectiveness of our algorithm. The complete block diagram of our segmentation technique is shown in Fig. 4, where $\boldsymbol{C}_{s}(\boldsymbol{x})$ is the segmented version of the color image $\boldsymbol{C}(\boldsymbol{x})$.

On the whole, it should be noticed that our technique performs pretty well. However, there exist some problems of oversegmentation in regions affected by highlights and shadows (see the images of the fruits, of the peppers, and of the yellow wall with the door). These limits are due to the fact that our segmentation algorithm is exclusively feature-based and that only color information is accounted for. In order to prevent oversegmentation, one should integrate both color and texture information and resort to physics-based techniques which account for the physical processes involved in the interaction of light with matter (e.g., see [35] and references therein). Improvement of the segmentation results could also be achieved by cascading our algorithm with a suitable post-processing stage. 


\section{Conclusions}

We have presented a new technique for low-level color image segmentation. It is based on two independent anisotropic diffusion processes: one applies to the chromatic information, conveniently embedded in a complex function; the second applies to the lightness information. The results of the two diffusions are separately segmented and their combination allows for the color image partitioning. We have reported some examples which confirm the good performance of our technique. Future research will be focused on the combination of color and feature information for image segmentation.

\section{Ackowledgements}

This work was supported in part by a University of California MICRO grant with matching supports from National Semiconductor Corp., Lucent Technologies, Tektronix Corp., and Xerox Corp.

\section{References}

[1] K.S. Fu and J.K. Mui, "A Survey on Image Segmentation," Pattern Recognition, Vol. 13, pp. 3-16, 1981.

[2] A. Rosenfeld and A. Kak, Digital Picture Processing, Vol. 2, $2^{\text {nd }}$ Ed., Academic Press, New York, NY, 1982.

[3] R.M. Haralick and L.G. Shapiro, "Image Segmentation Techniques," Comp. Vision, Graphics, and Image Proc., Vol. 29 , No. 1, pp. 100-132, Jan. 1985.

[4] N.R. Pal and S. K. Pal, "A Review on Image Segmentation Techniques," Pattern Recognition, Vol. 26, No. 9, pp. 1277-1294, 1993.

[5] Y. Rui, T.S. Huang, and S.-F. Chang, "Image Retrieval: Current Techniques, Promising Directions, and Open Issues," Journal of Visual Communication and Image Representation, Vol. 10, No. 1, pp. 39-62, Mar. 1999.

[6] W. Skarbek, and A. Koschan, "Colour image segmentation - A survey," Technical Report 94-32, Technical University Berlin, Oct. 1994.

[7] H.D. Cheng, X.H. Jiang, and Y. Sun, "A Survey on Color Image Segmentation," Proc. of the $6^{\text {th }}$ Int'l Conference on Fuzzy Theory and Technology, Research Triangle Park, NC, 23-28 Oct. 1998, Vol. 4, pp. 346-349. 
[8] L. Lucchese and S.K. Mitra, "Advances in Color Image Segmentation," Proc. of Globecom'99, Rio de Janeiro, Brazil, 5-9 Dec. 1999, pp. 2038-2044.

[9] P. Perona and J. Malik, "Scale Space and Edge Detection Using Anisotropic Diffusion," IEEE Trans. on PAMI, Vol. 12, No. 7, pp. 629-639, July 1990.

[10] B.M. ter Haar Romeny (Ed.), Geometry-Driven Diffusion in Computer Vision, Kluwer Academic Publishers, 1994.

[11] T. Dang, O. Jamet, and H. Maitre, "An Image Segmentation Technique Based on Edgepreserving Smoothing Filter and Anisotropic Diffusion," Proc. of IEEE Southwest Symp. on Image Analysis and Interpretation, pp. 65-69.

[12] J. Maeda, T. Iizawa, T. Ishizaka, C. Ishikawa, and Y. Suzuki, "Segmentation of Natural Images Using Anisotropic Diffusion and Linking of Boundary Edges," Pattern Recognition, Vol. 31, No. 12, pp. 1993-1999, 1998.

[13] E. Izquierdo and M. Ghanbari, M. "Using 3D Structure and Anisotropic Diffusion for Object Segmentation," Proc. the $7^{\text {th }}$ Int'l Congress on Image Processing and its Applications, Manchester, UK, 13-15 July 1999, Vol. 2, pp. 532-536.

[14] R. Whitaker and G. Gerig, "Vector-valued Diffusion," in Geometry-Driven Diffusion in Computer Vision, B.M. ter Haar Romeny (Ed.), pp. 93-134, July 1990.

[15] G. Sapiro and D.L. Ringach, "Anisotropic Diffusion of Multivalued Images with Application to Color Filtering," IEEE Trans. on Image Processing, Vol. 5, No. 11, pp. 1582-1586, Nov. 1996.

[16] L. Lucchese and S.K. Mitra, "Unsupervised Segmentation of Color Images Based on k-means Clustering in the Chromaticity Plane," in Proc. of IEEE Workshop on Content-based Access of Images and Video Libraries (CBAIVL'99), Fort Collins, CO, 22 June 1999, pp. 74-78.

[17] G. Wyszecki and W.S. Stiles, Color Science: Concepts and Methods, Quantitative Data and Formulae, Wiley, New York, NY, 1982.

[18] R.W. Massof and J.F. Bird, "A General Zone Theory of Color and Brightness Vision. I. Basaic Formulation," Journal of the Optical Society of America - A, Vol. 68, No. 11, pp. 1465-1471, Nov. 1978.

[19] O. Faugeras, "Digital Color Image Processing Within the Framework of a Human Visual Model," IEEE Trans. on Acoustics, Speech, and Signal Processing, Vol. ASSP-27, No. 4, pp. 380-393, Aug. 1979. 
[20] R. Gershon, "Aspects of Perception and Computation in Color Vision," Computer Vision, Graphics, and Image Processing, Vol. 32, pp. 244-277, 1985.

[21] R.W. Massof, "Color-Vision Theory and Linear Models of Color Vision," Color Research and Application, Vol. 10, No. 3, pp. 133-146, Fall 1985.

[22] S.L. Guth, "Model for Color Vision and Light Adaptation," Journal of the Optical Society of America - A, Vol. 8, No. 6, pp. 976-993, June 1991.

[23] S.G. Wolf, R. Ginosar, and Y.Y. Zeevi, "Spatio-Chromatic Image Enhancement Based on a Model of Human Visual Information Processing," Journal of Visual Communication and Image Representation, Vol. 9, No. 1, pp. 25-37, Mar. 1998.

[24] R.W.G. Hunt, The Reproduction of Colour, $5^{\text {th }}$ Ed., Fountain Press, Kingstone-upon-Thames, UK, 1995.

[25] R.W.G. Hunt, Measuring Colour, $2^{\text {nd }}$ Ed., Ellis Horwood Ltd. Publ., Chichester, England, 1987.

[26] A. McCabe, G. West, and T. Caelli, "Filter Techniques for Complex Spatio-Chromatic Image Processing," Proc. of ICIP98, Vol. II, pp. 742-746.

[27] S.J. Sangwine and R.E.N. Horne, Eds., The Colour Image Processing Handbook, Chapman \& Hall, London UK, 1998.

[28] P. Perona, "Orientation Diffusions," Proc. of IEEE Trans. on Image Processing, Vol. 7, No. 3, pp. 457-467, Mar. 1998.

[29] B. Tang and G. Sapiro, "Chromaticity Diffusion," Proc. of Int'l Conf. on Image Processing (ICIP 2000), Vancouver, Canada, 10-13 Sept. 2000, Vol. II, pp. 784-787.

[30] L. Lucchese and S.K. Mitra, "Unsupervised Low-Frequency Driven Segmentation of Color Images," Proc. of Int'l Conf. on Image Processing (ICIP'99), Kobe, Japan, 25-28 Oct. 1999, Vol. III, pp. 240-244.

[31] P.S. Heckbert, "Color Image Quantization for Frame Buffer Display", Computer Graphics, Vol. 16, No. 3, pp. 297-303, 1982.

[32] M.T. Orchard and C. Bouman, "Color Quantization of Images", IEEE Trans. on Signal Processing, Vol. 35, No. 12, pp. 2677-2690, Dec. 1991.

[33] H. Stark and J.W. Woods, Probability, Random Processes, and Estimation Theory for Engineers, $2^{\text {nd }}$ Ed., Prentice Hall, Englewood Cliffs, NJ, 1994. 
[34] M.R. Anderberg, Cluster Analysis for Applications, Academic Press, New York, NY, 1973.

[35] B.A. Maxwell and S.A. Shafer, "Physics-Based Segmentation of Complex Object Using Multiple Hypotheses of Image Formation," Computer Vision and Image Understanding, Vol. 65, No. 2, pp 269-295, Feb. 1997. 
(a)

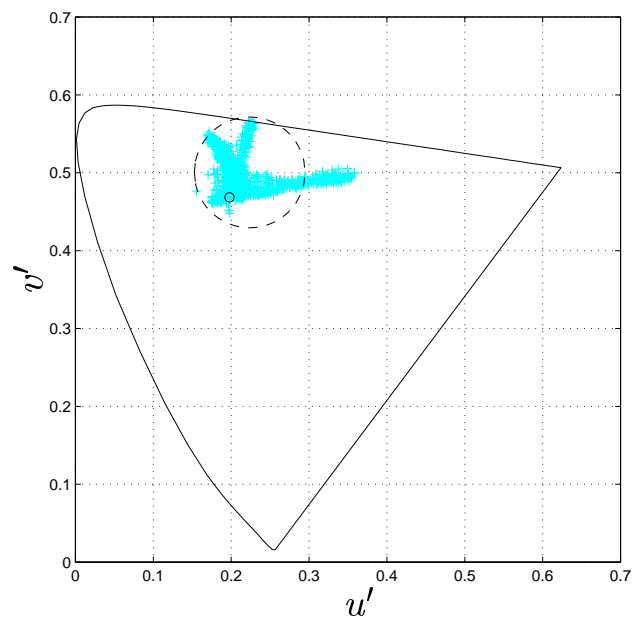

(b)

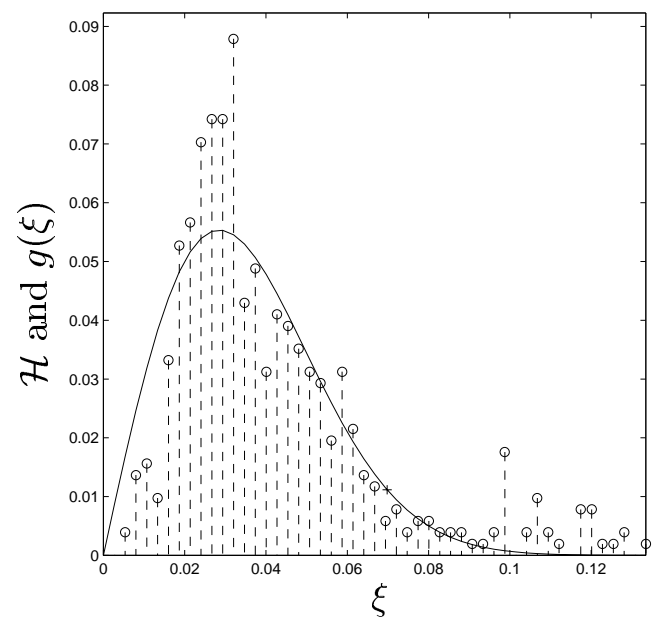

Figure 1: (a) $u^{\prime} v^{\prime}$ chromaticity diagram relative to the image of the parrots of Fig. 7; the small circle is the reference white while the large dashdotted circle is centered at the baricenter of the chromaticities and contains the $95 \%$ of the data (see Section 3.3). (b) Frequency histogram $\mathcal{H}$ (dashdotted bars) and Rayleigh pdf $g(\xi)$ (solid line) relative to the chromaticities of Fig. 1 (a).

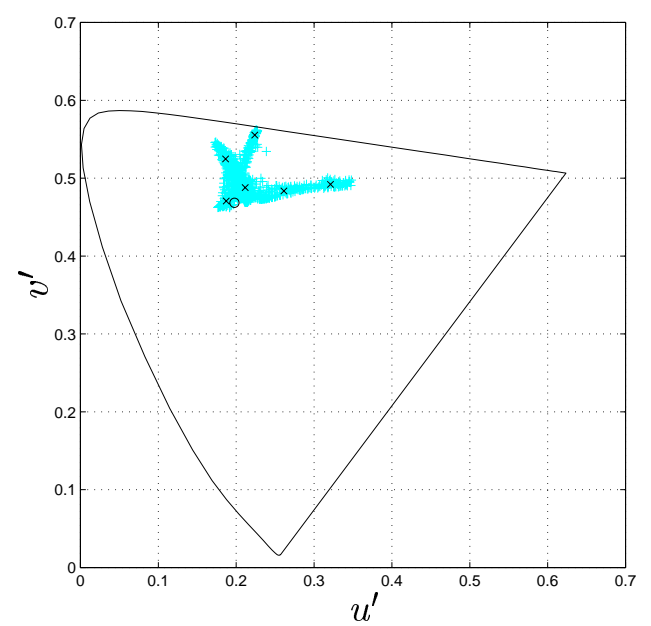

Figure 2: $u^{\prime} v^{\prime}$ chromaticity diagram relative to the image of the parrots of Fig. 7 after diffusion and color quantization. The crosses denote the clusters $\boldsymbol{\omega}(n)=\boldsymbol{\sigma}_{c}(n) \exp \left(j \boldsymbol{\vartheta}_{c}(n)\right), n=1, \ldots, N_{\omega}=6$ (see Section 3.4). 

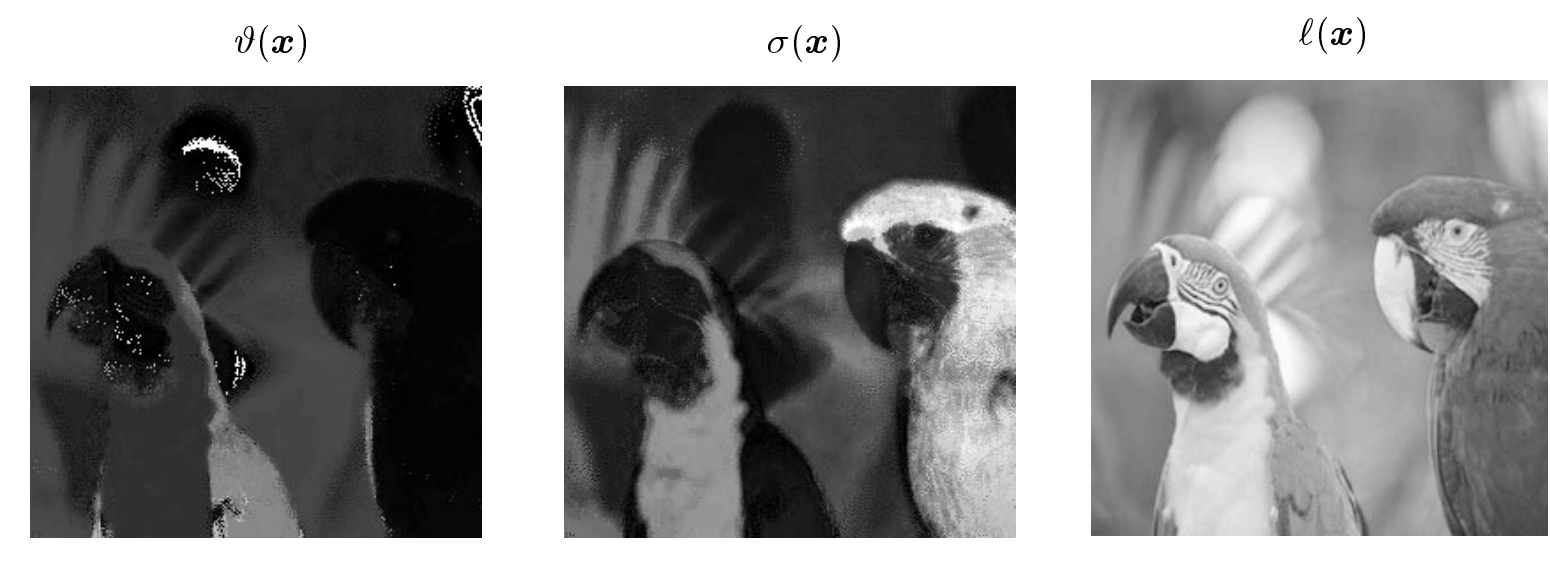

$\vartheta_{d}(\boldsymbol{x})$
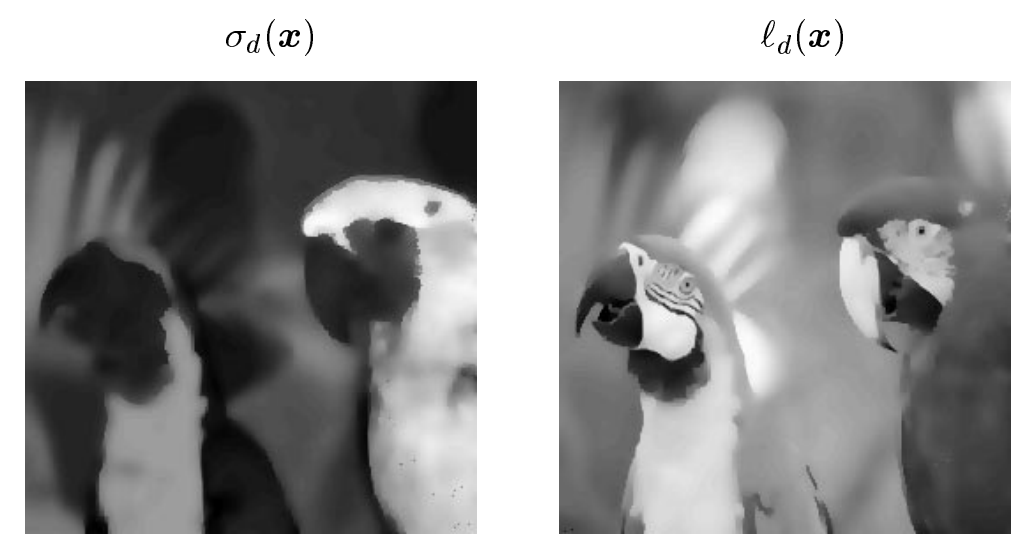

$\vartheta_{s}(\boldsymbol{x})$

$$
\sigma_{s}(\boldsymbol{x})
$$

$$
\ell_{s}(\boldsymbol{x})
$$
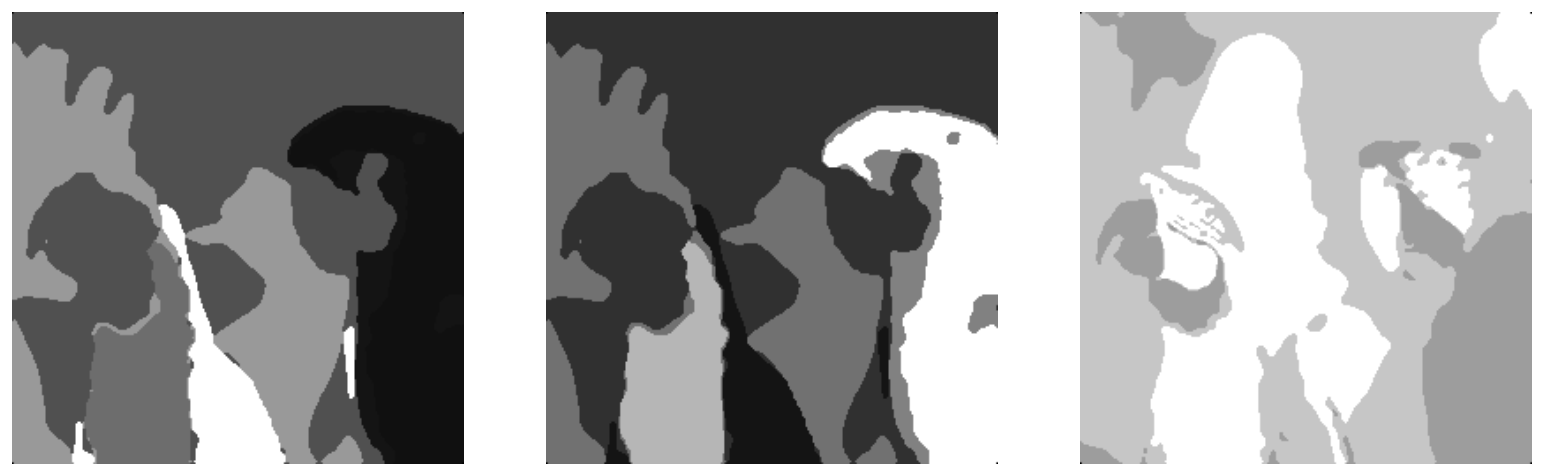

Figure 3: First row: Original hue $\vartheta(\boldsymbol{x})$, saturation $\sigma(\boldsymbol{x})$, and lightness $\ell(\boldsymbol{x})$ (from left to right). Second row: Diffused hue $\vartheta_{d}(\boldsymbol{x})$, saturation $\sigma_{d}(\boldsymbol{x})$, and lightness $\ell_{d}(\boldsymbol{x})$. Third row: Segmented hue $\vartheta_{s}(\boldsymbol{x})$, saturation $\sigma_{s}(\boldsymbol{x})$, and lightness $\ell_{s}(\boldsymbol{x})$. 


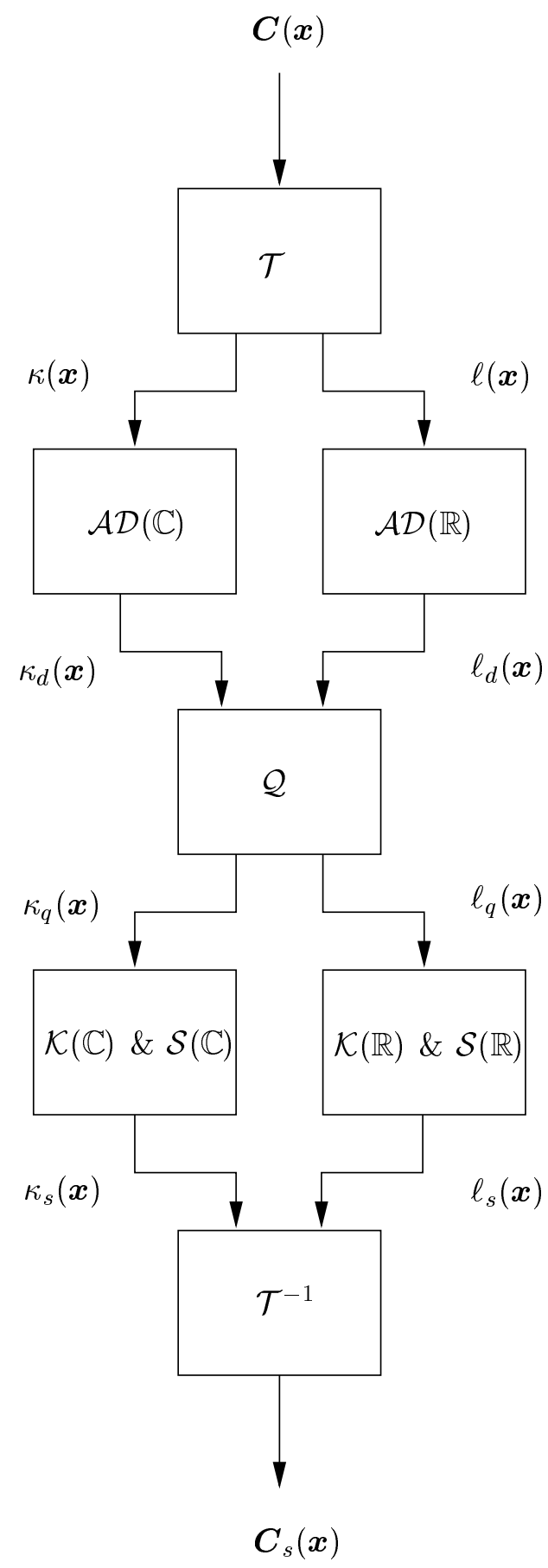

Figure 4: Block diagram of the segmentation algorithm. Legend: $\boldsymbol{C}(\boldsymbol{x}):=$ color image to be segmented; $\mathcal{T}:=$ color transformation from $R G B$ coordinates to complex chromatic channel and lightness channel; $\mathcal{T}^{-1}:=$ inverse transformation of $\mathcal{T} ; \mathcal{A D}(\mathbb{C}):=$ anisotropic diffusion of $\kappa(\boldsymbol{x}) ; \mathcal{A D}(\mathbb{R}):=$ anisotropic diffusion of $\ell(\boldsymbol{x}) ; \mathcal{Q}:=$ quantization; $\mathcal{K}(\mathbb{C}):=$ k-means clustering in $\mathbb{C} ; \mathcal{K}(\mathbb{R}):=$ k-means clustering in $\mathbb{R} ; \mathcal{S}(\mathbb{C}):=$ segmentation of the chromatic channel; $\mathcal{S}(\mathbb{R}):=$ segmentation of the achromatic channel; $\boldsymbol{C}_{s}(\boldsymbol{x}):=$ segmented image. 
(a)
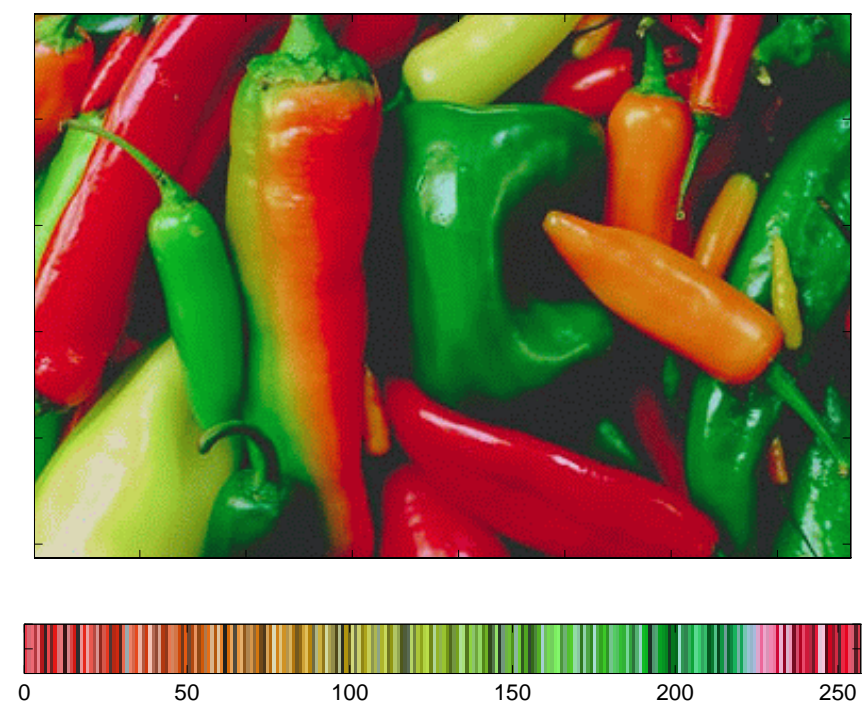

(b)

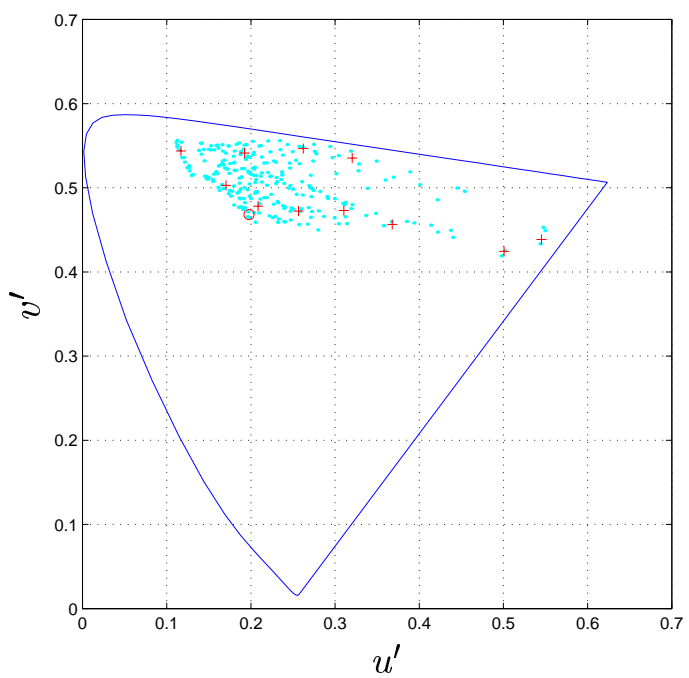

(c)

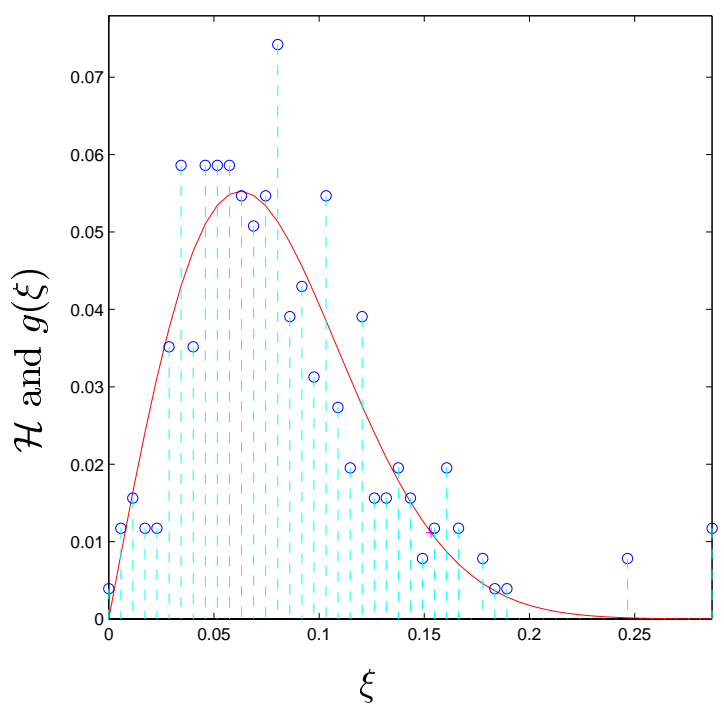

Figure 5: a) A color image with its color palette; b) Its $u^{\prime} v^{\prime}$ chromaticity diagram with clusters (red +'s) found by the algorithm of Section 3.4; c) Relative histogram $\mathcal{H}$ (stemmed plot) and Rayleigh pdf $g(\xi)$ (red solid line). 
(a)
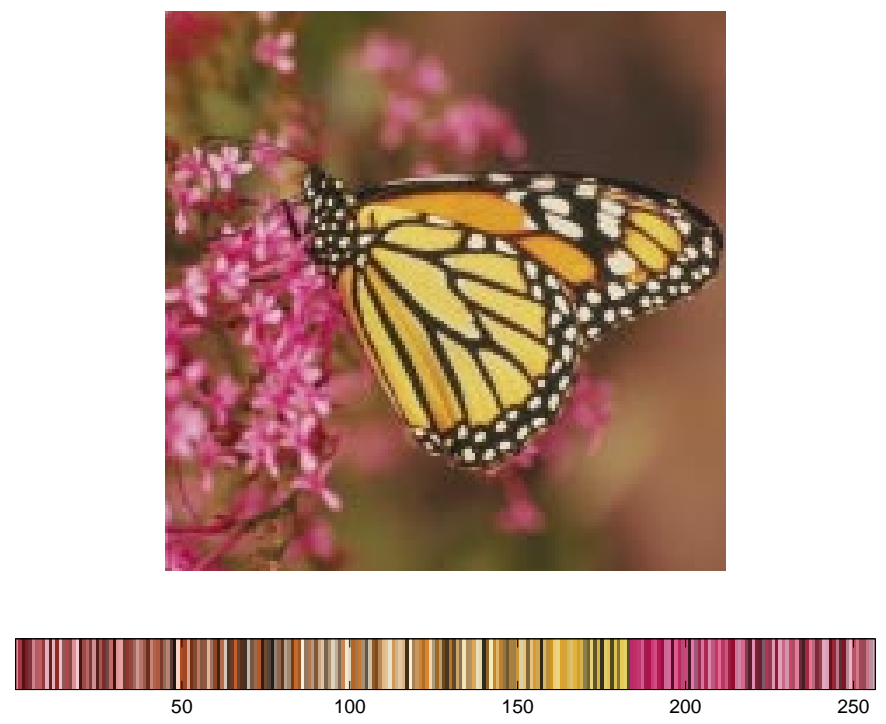

(b)

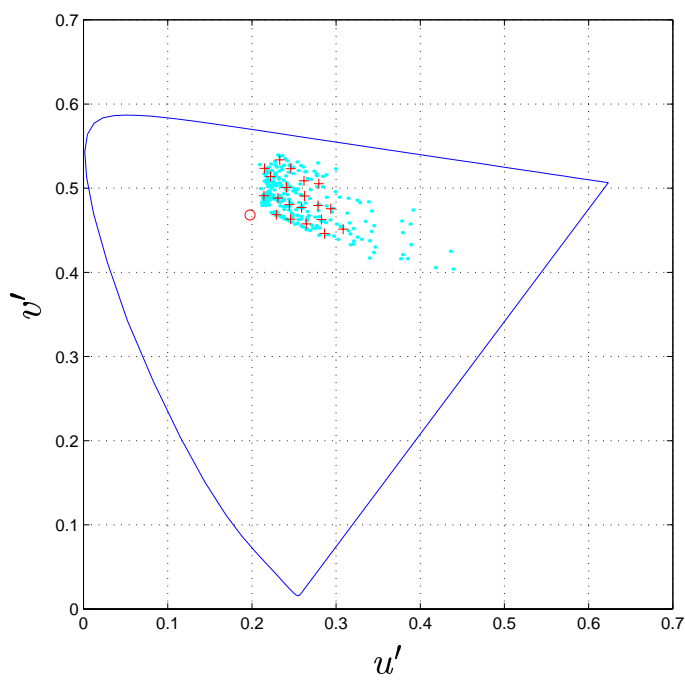

(c)

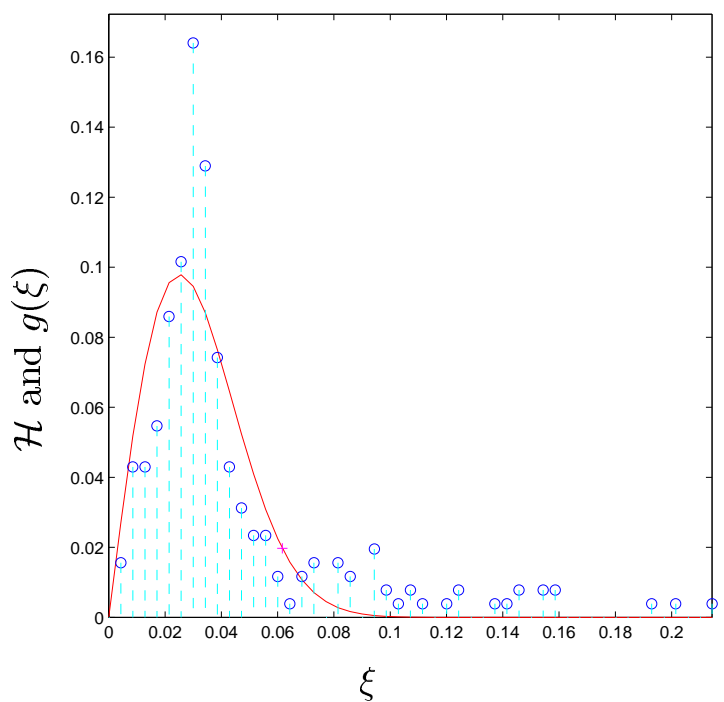

Figure 6: a) A color image with its color palette; b) Its $u^{\prime} v^{\prime}$ chromaticity diagram with clusters (red +'s) found by the algorithm of Section 3.4; c) Relative histogram $\mathcal{H}$ (stemmed plot) and Rayleigh pdf $g(\xi)$ (red solid line). 
original images
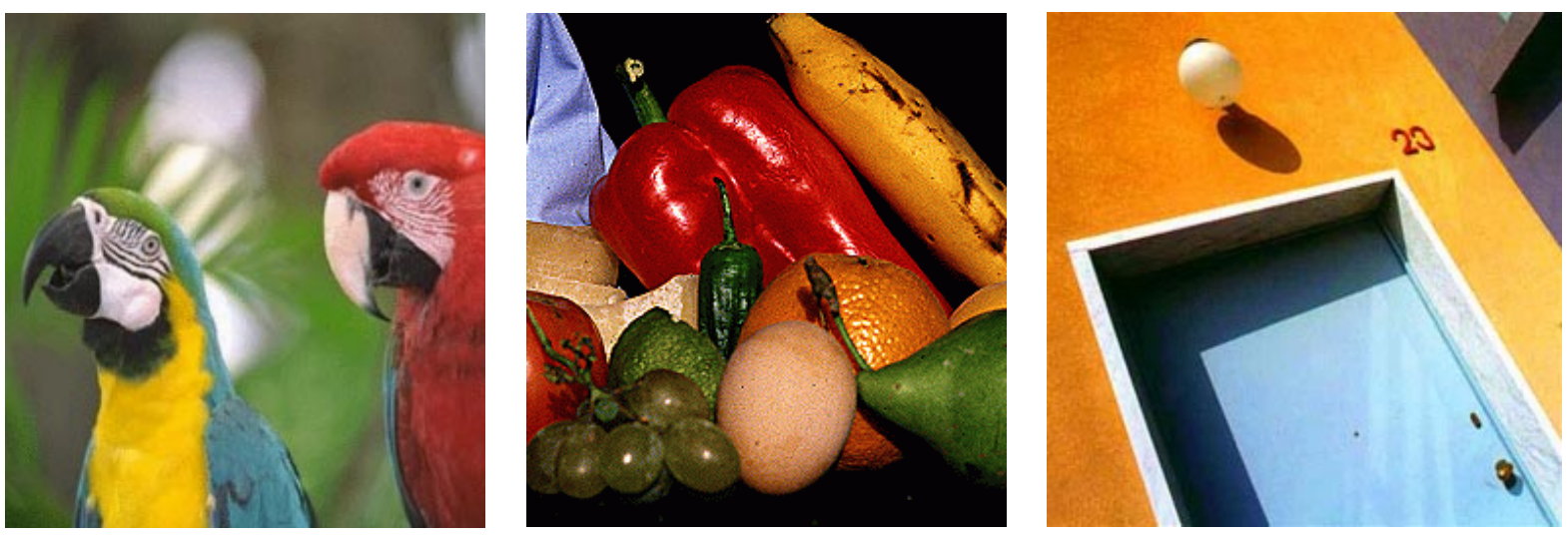

segmented images
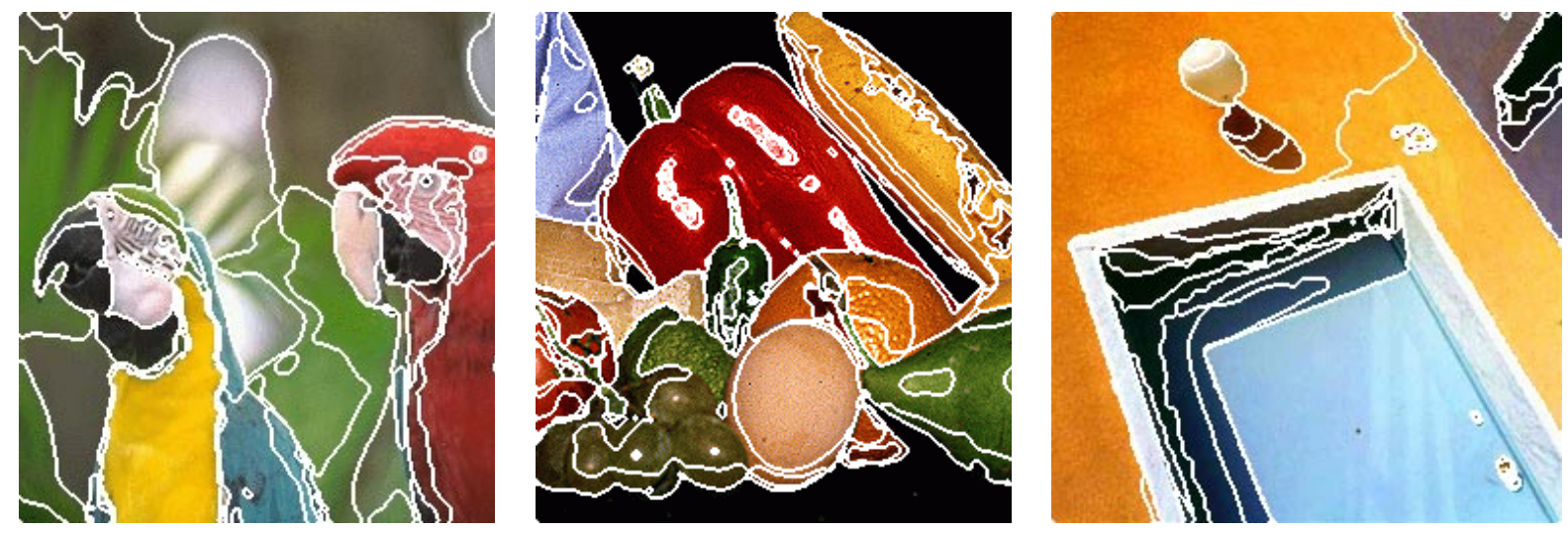

Figure 7: Some examples of segmentation. 
original images
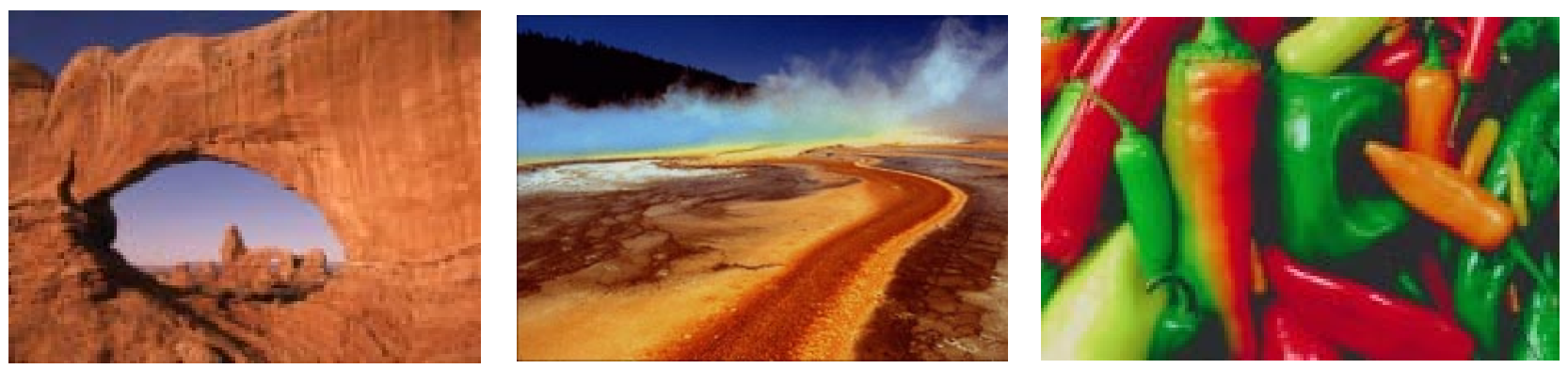

segmented images
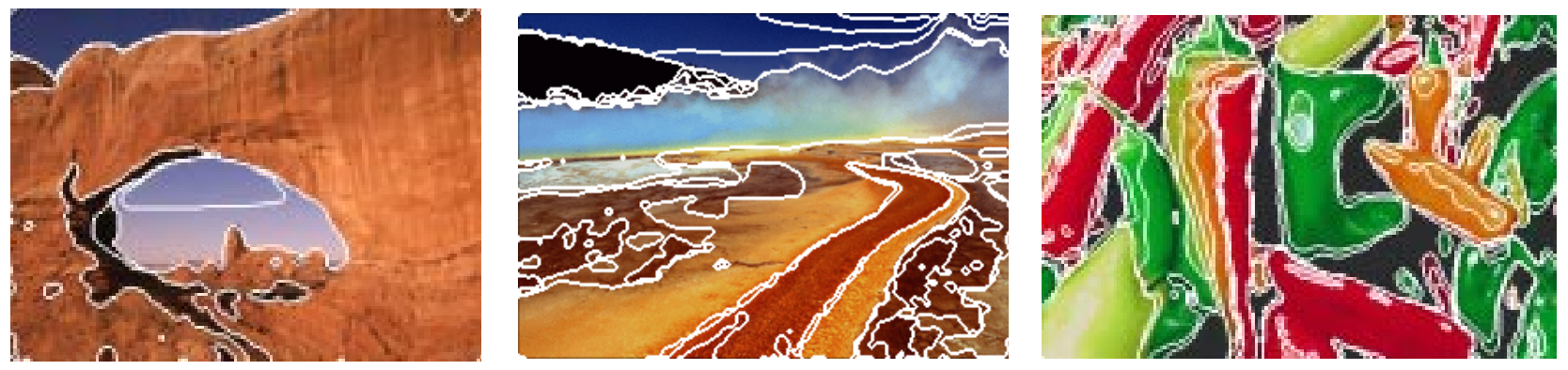

Figure 8: Some other examples of segmentation. 